\title{
Proyectos pedagógicos productivos una herramienta para el proceso de enseñanza-aprendizaje
}

Productive pedagogical projects a tool for the teaching-learning process

\section{Projetos pedagógicos produtivos uma ferramenta para o processo de ensino-aprendizagem}

\author{
Becerra Guavita Jhon Jairo ${ }^{1}$, Herrera Gutiérrez Nataly ${ }^{1}$ y \\ Rodríguez Rodríguez Mónica del Pilar² \\ ${ }^{1}$ Licenciados en Producción Agropecuaria, Universidad de los Llanos y \\ ${ }^{2}$ LPA, Esp. MSc. Docente Universidad de los Llanos \\ mrodriguez@unillanos.edu.co
}

Recibido 14 de Marzo 2018, Aceptado 22 de Octubre 2018

\section{RESUMEN}

El objetivo de este trabajo fue caracterizar y analizar los proyectos pedagógicos productivos (PPP) desarrollados por los estudiantes de la práctica profesional docente del programa de Licenciatura en Producción Agropecuaria, durante un periodo de tres años, con el fin de evidenciar el fortalecimiento de la modalidad agropecuaria en las distintas instituciones educativas ubicadas en el departamento del Meta, Colombia. Para la recolección de la información se consultaron bases de datos de cinco instituciones educativas de la zona, se diseñaron encuestas que fueron aplicadas a estudiantes, asesores de prácticas, padres de familia y estudiantes en práctica pedagógica (EPP) de la Universidad de los Llanos; también se realizaron reuniones con los EPP donde presentaron los proyectos en los cuales participaron, allí se evidenciaron los factores que incidieron en el impacto generado por estos proyectos en las distintas instituciones educativas, y así mismo se analizó su beneficio en la formación de los EPP tanto a nivel profesional como personal e incluso en sus familias. Se concluye que estas acciones curriculares en la mayoría de los casos fortalecen contenidos temáticos de la modalidad agropecuaria, por lo tanto, su participación fue dinámica para el éxito de los PPP, los cuales incidieron en el mejoramiento de la calidad de vida de algunas familias que participaron, 
permitiendo ofrecer alternativas de seguridad alimentaria y el fortalecimiento de los procesos productivos en las instituciones. Así mismo, los practicantes manifestaron que el ejercicio de la práctica profesional docente permite desarrollar destrezas, habilidades, conocimientos académicos, y personales que son aplicados en el diseño, ejecución y evaluación de los PPP, lo cual es una preparación para abordar los retos del campo laboral.

Palabras clave: Práctica docente, comunidad educativa, seguridad alimentaria, agricultura familiar.

\section{ABSTRACT}

The objective of this work was to characterize and analyze the productive pedagogical projects (PPP) developed by the students of the professional teaching practice of the Bachelor program in Agricultural Production, during a period of three years in order to demonstrate the strengthening of the agricultural modality in the different educational institutions located in the department of Meta, Colombia. For the collection of information, databases of five educational institutions in the area were consulted, surveys were designed that were applied to students, practice advisors, parents and students in pedagogical practice (SPP) of the Universidad de los Llanos; meetings were also held with the SPP where they presented the projects in which they participated, there were evidenced the factors that influenced the impact generated by these projects in the different educational institutions, and likewise, was analyzed their benefit in the formation of SPP, both professionally and personally, and even in their families. It is concluded that these curricular actions in most cases strengthen thematic contents of the agricultural modality, therefore, their participation was dynamic for the success of the PPP, which affected the improvement of the quality of life of some families that participated, allowing to offer alternatives of food security and the strengthening of the productive processes in the institutions. Likewise, the practitioners stated that the exercise of professional teaching practice allows to develop skills, abilities, academic knowledge, and personal that are applied in the design, execution and evaluation of PPP, which is a preparation to address the challenges of the workplace. 
Keywords: Teaching practice, educational community, food security, family farming.

\section{RESUMO}

O objetivo deste trabalho foi caracterizar e analisar os projetos pedagógicos produtivos (PPP) desenvolvidos pelos alunos da prática docente profissional do programa de Bacharel em Produção Agrícola, durante um período de três anos, a fim de demonstrar o fortalecimento da modalidade agrícola nas diferentes instituições educacionais localizadas no departamento de Meta, Colômbia. Para a coleta de informações foram consultadas bases de dados de cinco instituições de ensino da área, foram elaboradas questionários que foram aplicadas a estudantes, orientadores de prática, pais de família e estudantes em prática pedagógica (EPP) da Universidad de los Llanos; também foram realizadas reuniões com o EPP, onde apresentaram os projetos dos quais participaram, e os fatores que afetaram o impacto gerado por esses projetos nas diferentes instituições de ensino foram evidenciados, e da mesma forma, foi analisado seu benefício na formação de EPP, tanto profissional quanto pessoalmente, e até mesmo em suas famílias. Conclui-se que essas ações curriculares, na maioria dos casos, fortalecem conteúdos temáticos da modalidade agrícola, portanto, sua participação foi dinâmica para o sucesso da PPP, que afetou a melhoria da qualidade de vida de algumas famílias que participaram, permitindo oferecer alternativas de segurança alimentar e o fortalecimento dos processos produtivos nas instituições. Da mesma forma, os praticantes afirmaram que o exercício da prática profissional docente permite desenvolver habilidades, habilidades, conhecimento acadêmico e pessoal que é aplicado na concepção, execução e avaliação de PPP, que é uma preparação para enfrentar os desafios do campo de trabalho.

Palavras-chave: Prática docente, comunidade educativa, segurança alimentar, agricultura familiar.

\section{INTRODUCCIÓN}

Los proyectos pedagógicos productivos (PPP) pueden estar orientados al diseño y elaboración de un producto, al aprovechamiento del material didáctico mediante el 
dominio de alguna técnica o tecnología, solución de un caso particular de la vida académica, social, política o económica y en general, al desarrollo de intereses de los educandos que promuevan su espíritu investigativo, y cualquier otro propósito que cumpla los fines y objetivos en el proyecto educativo, puesto que la meta de la formación de los profesionales no es solo enseñarles a los estudiantes en prácticas laborales cómo ejercer su profesión, es más importante que ellos aprendan a dar continuidad en contextos laborales diversos (MEN, 2012a).

La planificación de proyectos pedagógicos es el resultado de un proceso de búsqueda ante el imperativo de acercar la escuela a las experiencias de vida, en este sentido, el proyecto se asume como una construcción en la que es necesaria la participación del maestro, alumnos, representantes y otros miembros de la sociedad; como es una construcción, los criterios para su formulación y desarrollo no se pueden imponer desde fuera, porque se generan a partir de la discusión y reflexión en el aula u otros espacios donde se realiza actividad pedagógica (Ortíz, 2013).

A través de esta concepción de la planificación, en proyectos de aula se logra mayor congruencia con los aportes acerca del conocimiento y el aprendizaje, a la vez que está a tono con las aspiraciones filosóficas y las bases legales que todavía sustentan nuestra educación. Además de lo anterior, la planificación por proyectos contribuye al desarrollo del docente como intelectual, pues lo inquieta y conduce a una constante actualización de sus saberes en la construcción de herramientas cognoscitivas (Becerra, 2004).

Cuando se inició todo este proceso de la necesidad de cambio, la planificación de la enseñanza se tornó en una actividad difícil surgiendo entonces la necesidad urgente de experimentar en la práctica pedagógica metodologías más pertinentes y convenientes determinando su viabilidad para contribuir con la sistematización de las mismas (Moreno et al., 2010).

Los proyectos pedagógicos productivos (PPP) son una herramienta que se puede utilizar en la transformación participativa y comunitaria de la educación rural a partir 
del diseño y puesta en marcha de proyectos agropecuarios pertinentes y sostenibles. Puede considerarse como dedicación por parte de la comunidad educativa a la producción de determinados bienes o servicios para acrecentar sus beneficios económicos sociales y culturales. En este contexto los PPP tienen por beneficiarios a la comunidad educativa en la localidad donde se realicen, en estos se deben dimensionar las circunstancias particulares de la zona y ser pertinentes a las necesidades de las mismas, valorando las potencialidades culturales, deportivas y artísticas de la comunidad educativa, sin dejar nunca de lado, los saberes locales. En los PPP se proponen alternativas económicas para desarrollar en un proceso integral de formación académica aplicando conceptos mediante actividades prácticas, que contribuyan al fortalecimiento y la construcción de alternativas de vida, que posibiliten la convivencia y supervivencia de las comunidades y su cultura en reciprocidad y equilibrio armónico con los procesos naturales, partiendo de la identificación e implementación pedagógica de alternativas para mejorar o cambiar las formas de producción y el uso de los recursos, y el mejoramiento de la calidad de vida teniendo como fundamento la sustentabilidad social, económica, cultural y ambiental de las comunidades rurales (GVC y UV, 2013).

El principal reto es propender por la construcción de alternativas económicamente viables, ecológicamente respetuosas, culturalmente aceptables y socialmente justas para las comunidades rurales, proponiendo así alternativas de desarrollo sin destruir los recursos naturales y sin transformar las unidades familiares campesinas en unidades especializadas y asalariadas (MEN, 2012b).

Los componentes de los PPP, como su nombre lo indica incluye elementos pedagógicos y productivos, concebidos para que funcionen de manera interdependiente y complementaria; esto quiere decir que, si uno de ellos no es considerado, o existe un desbalance en su énfasis, se corre el riesgo de limitar su alcance o errar en su concepción. Además, en los PPP se analizan y planean acciones tales como la identificación, priorización y análisis de los impactos ambientales sobre el capital natural; la toma de decisiones para la intervención ambiental sostenible; la formación para la responsabilidad ambiental; y la 
priorización del bienestar de los proyectos de vida, personales, laborales, profesionales y comunitarios (MEN, 2010b; Ortíz, 2013). Esto permite a los docentes tener una oportunidad para transformar y desarrollar sus prácticas pedagógicas e interdisciplinares al establecer relaciones entre el conocimiento escolar, la vida cotidiana y el mundo productivo. Igualmente, consiguen hacer pertinentes y flexibles los conocimientos en el proceso educativo (MEN, 2010a).

Un proyecto en su dimensión educativa, es el resultado de una metodología de trabajo interdisciplinar donde la enseñanza y el aprendizaje están guiados por los conceptos, procedimientos, habilidades y actitudes que los estudiantes vienen desarrollando en un escenario escolar que promueve una formación para la vida, e interacción permanente con el mundo social, cultural y deportivo. A través de la metodología por proyectos se da respuesta a situaciones o aspectos que pueden ser potenciados en contextos productivos específicos, permitiendo así transferir el conocimiento escolar a situaciones reales donde los estudiantes toman decisiones de manera autónoma, mientras aprenden a trabajar de forma colaborativa.

Además, el componente pedagógico está relacionado con la construcción del aprendizaje significativo, mediante el desarrollo de competencias en estudiantes y procesos de enseñanza, que teniendo en cuenta la flexibilidad en tiempos y espacios vinculan los diferentes aportes de estudiantes, docentes, directivos, padres, comunidad, instituciones técnicas y sector productivo. Este componente propone dejar atrás el trabajo disciplinar que se basa en la reproducción de conocimientos, la fragmentación de la realidad y el desconocimiento de los contextos y saberes previos construidos por los estudiantes (FMM, 2010).

El componente productivo se refiere a la productividad entendida en términos económicos, intelectuales y actitudinales lo que implica actividades diversas en agricultura, artesanía, industria, servicios, cultura, recreación y otros, además conlleva a una relación permanente con los contextos locales, regionales, nacionales y globales, observando las formas de producción y comercialización, lo que significa mayor correspondencia con empresas industriales y de distribución, lo cual permite prácticas de gestión innovadoras. En el sector agrario se promueve el 
desarrollo de acciones para la conservación y cuidado del medio ambiente, como la agricultura orgánica y ecológica, la preservación de bosques, fuentes de agua, y la transformación de productos agropecuarios como la elaboración de mermeladas, embutidos, bocadillos, arequipe y otros productos (Pacheco et al., 2009).

Hay que recordar que la cultura del emprendimiento fortalece los conocimientos, habilidades y actitudes que permiten a los estudiantes, docentes y comunidades construir grupos de trabajo para abordar mejor las problemáticas sociales. Igualmente, los proyectos pedagógicos productivos permiten involucrar de manera proactiva al mundo económico y el desarrollo social, promoviendo autonomía, autoestima, sentido de pertenencia, creatividad, asociatividad y solidaridad (Martínez y Esteban, 2005).

\section{INSTITUCIONES EDUCATIVAS CARACTERIZADAS}

La ley 115 de 1994, decretada por el Congreso de la Republica de Colombia, en su artículo 13, establece que es objetivo primordial de todos y cada uno de los niveles educativos el desarrollo integral de los educandos mediante acciones estructuradas encaminadas a: 1) Formar la personalidad y la capacidad de asumir con responsabilidad y autonomía sus derechos y deberes; 2) Proporcionar una sólida formación ética y moral, y fomentar la práctica del respeto a los derechos humanos; 3) Fomentar en la institución educativa, prácticas democráticas para el aprendizaje de los principios y valores de la participación y organización ciudadana, y estimular la autonomía y la responsabilidad; 4) Desarrollar una sana sexualidad que promueva el conocimiento de sí mismo y la autoestima, la construcción de la identidad sexual dentro del respeto por la equidad de los sexos, la afectividad, el respeto mutuo y prepararse para una vida familiar armónica y responsable; 5) Crear y fomentar una conciencia de solidaridad internacional; 6) Desarrollar acciones de orientación escolar, profesional y ocupacional; 7) Formar una conciencia educativa para el esfuerzo y el trabajo, y 8) Fomentar el interés y el respeto por la identidad cultural de los grupos étnicos. 
Considerando estos objetivos plasmados en la ley de educación en Colombia, el objetivo de este trabajo fue caracterizar los proyectos pedagógicos productivos desarrollados en la práctica profesional docente del programa de Licenciatura en Producción Agropecuaria (LPA) de la Universidad de los Llanos, analizando evidencias de actores involucrados en el diseño, ejecución y evaluación de los mismos como lo son estudiantes, asesores de práctica, padres de familia, comunidades y practicantes de LPA, quienes trabajaron en el área de influencia los PPP en las siguientes cinco instituciones educativas, ubicadas en los municipios de Puerto López, San Martín, Acacias, San Juan de Arama y Lejanías, todos ubicados en el Departamento del Meta.

\section{Institución Educativa Triétnica Yaaliakeisy}

Se encuentra ubicada en Puerto López, siendo su principal actividad económica la agricultura, ganadería, pesca, comercio, transporte, prestación de servicios públicos y de manera incipiente la agroindustria. Se ha desarrollado el turismo porque es el centro geográfico de Colombia, ubicado en el Alto de Menegua donde se encuentra el pozo de los deseos, adicionalmente en los últimos años la cultura hace parte fundamental del desarrollo de Puerto López puesto que es muy común escuchar la música llanera propia de la región (ALPL, 2017).

\section{Institución Educativa Iraca}

Se localiza en el municipio de San Martín, ha sido considerada la mejor institución educativa pública del departamento gracias a los excelentes resultados logrados en las pruebas del ICFES alcanzados por sus estudiantes de grado once. Tiene una granja donde se desarrollan proyectos productivos de palma de aceite, agroturismo, piscicultura, caucho, árboles maderables, conservación de ganado Sanmartinero, ganadería doble propósito, especies menores, cítricos y zoocría de especies nativas como el chigüiro (ALSM, 2004).

San Martín posee diferentes ecosistemas que lo convierten en una unidad ecológica, en la cual diferentes grupos de organismos interactúan con el ambiente; la dinámica de estos ecosistemas en algunos casos se encuentra alterada por el 
desequilibrio presente en la cadena trófica debido a la pérdida de la cobertura vegetal, que se ve afectada por la intervención antrópica, del mismo modo la fauna sufre procesos de desplazamiento hacia áreas que le ofrezcan mejores condiciones para su existencia. Con relación a sus actividades económicas, el sector ganadero dispone del $63 \%$ de la superficie total del municipio mientras que el agrícola ocupa el $1.74 \%$, prevalece la ganadería extensiva, aunque se pueden encontrar cultivos de palma africana, arroz patilla, yuca, cítricos y plátano. Se realizan actividades culturales como el Festival Internacional Folclórico y Turístico del Llano y la presentación de las famosas cuadrillas de San Martín, que son un original ballet ecuestre, de gran calidad artística, argumento, ritmo y colorido, ejecutadas por cuarenta y ocho jinetes, distribuidos en cuatro grupos de doce chalanes cada uno (ALSM, 2012).

\section{Institución Educativa Campestre San José}

Ubicada en la vereda de su mismo nombre, municipio de Acacias Meta, a siete km del casco urbano. La Alcaldía concedió un terreno ubicado en la vereda de Rancho Grande a 1.400 metros del Colegio, con un área de cuatro hectáreas para que se realicen allí las prácticas propias de la modalidad. En Acacias el principal sector económico es el agropecuario, la industria es pequeña predominando los procesos artesanales, aunque la minería y la explotación petrolera han adquirido últimamente gran importancia. El sector terciario se evidencia en un comercio fuerte en la zona urbana con énfasis en la venta de productos terminados más que de servicios. Algunas actividades culturales son: Encuentros de Colonias y Festival del Retorno (ALA, 2016).

\section{Institución Educativa Manacal}

Ubicada en la vereda Buenos Aires, Municipio de San Juan de Arama, localizado cerca de la sierra de la Macarena, por lo tanto, su paisaje cuenta con hermosos valles y grandes sabanas de la región del rio Ariari, que cuenta con vegas de alta fertilidad las cuales contribuyen considerablemente al desarrollo de esta región, por 
lo tanto, la principal actividad económica en la zona es la producción ganadera y agrícola (CMSJA, 2016).

\section{Institución Educativa de Lejanías}

Recibe el nombre del mismo del municipio, cuenta con una gran diversidad climática, variedad de fauna, paisajes y una inmensa hidrografía. El uso del suelo se presenta por el mayor o menor grado de desarrollo del sector agropecuario, principalmente el eje de crecimiento económico ha sido el agrícola que tiene mayor relevancia que la ganadería. Los cultivos más representativos son: cítricos, guayaba pera, cacao, café, fríjol, maracuyá, piña, mora, aguacate, soya, papaya, lulo, plátano, caña panelera, maíz, arazá y noni, y en el sector pecuario se destaca la piscicultura y avicultura (ALL, 2016).

\section{METODOLOGÍA}

Este proyecto es de tipo descriptivo con enfoque pedagógico y social, el cual fue realizado con las comunidades educativas de los siguientes colegios agropecuarios, ya mencionados: Triétnica Yaaliakeisy (Puerto López), Iraca (san Martín), San José (Acacias), Macanal (San Juan de Arama) e Institución Educativa de Lejanías (Lejanías). Entendida la investigación descriptiva desde la perspectiva de Sabino, (2014) como aquella que trabaja sobre realidades de hechos, y su característica fundamental es la de presentar una interpretación correcta; para la investigación descriptiva, su preocupación primordial radica en descubrir algunas características fundamentales de conjuntos homogéneos de fenómenos, utilizando criterios sistemáticos que permitan poner de manifiesto su estructura o comportamiento, de tal manera que se pueden obtener las notas que caracterizan a la realidad estudiada. Esta investigación también tuvo un enfoque social, teniendo en cuenta que el desarrollo de proyectos pedagógicos productivos se ha constituido en la herramienta didáctica, a partir de la cual se intervienen las comunidades educativas con orientación hacia el mejoramiento de la calidad de vida y de los sistemas de producción. 
La población estuvo conformada por 48 estudiantes de la Universidad de los Llanos pertenecientes al programa Licenciatura en Producción Agropecuaria (LPA), quienes realizaron su práctica profesional docente durante el periodo comprendido entre los tres años evaluados en las mencionadas instituciones educativas, además participaron: asesores práctica, estudiantes de las instituciones educativas y padres de familia.

Para la recolección de la información se consultaron las bases de datos de las instituciones educativas para la identificación de los PPP y a partir de allí se elaboró una encuesta que fue aplicada a los estudiantes, asesores, practicantes y padres de familia. También se realizaron visitas para hacer observaciones directas en los escenarios educativos con el fin de determinar características que pudieran aportar elementos para establecer el impacto de los PPP desarrollados durante un periodo de tres años, que fue el tiempo evaluado. Con el fin de validar y ampliar la información sobre el impacto de los PPP desarrollados, se conformó un grupo focal donde se reunían profesores de las instituciones educativas donde los estudiantes de la práctica pedagógica (EPP) presentaron los proyectos en los cuales participaron y las etapas del proceso que ellos alcanzaban a ejecutar con éxito.

Este proyecto fue ejecutado según el seguimiento y la evaluación de estudios realizados por los miembros pertenecientes al proyecto institucional "Impacto de los proyectos pedagógicos productivos desarrollados en la práctica profesional docente del programa LPA de UNILLANOS", en su contexto de aplicación durante el periodo evaluado, esto con el fin de contribuir con el mejoramiento de las propuestas relacionadas con la ejecución de los PPP por parte de los practicantes.

Teniendo en cuenta los objetivos específicos planteados se organizaron las siguientes actividades: 1) Visita de acercamiento a las instituciones educativas y presentación del proyecto por parte del equipo investigador, lo cual se realizó durante cinco meses; 2) Aplicación de la encuesta a los asesores de práctica, estudiantes de las instituciones educativas y padres de familia; 3) Análisis de la información y elaboración de matrices y rejillas; 4) Devolución de resultados a las comunidades para validar la información; y 5) Validación de la información a través 
del grupo focal con practicantes y docentes de las instituciones educativas, (Figura 1).

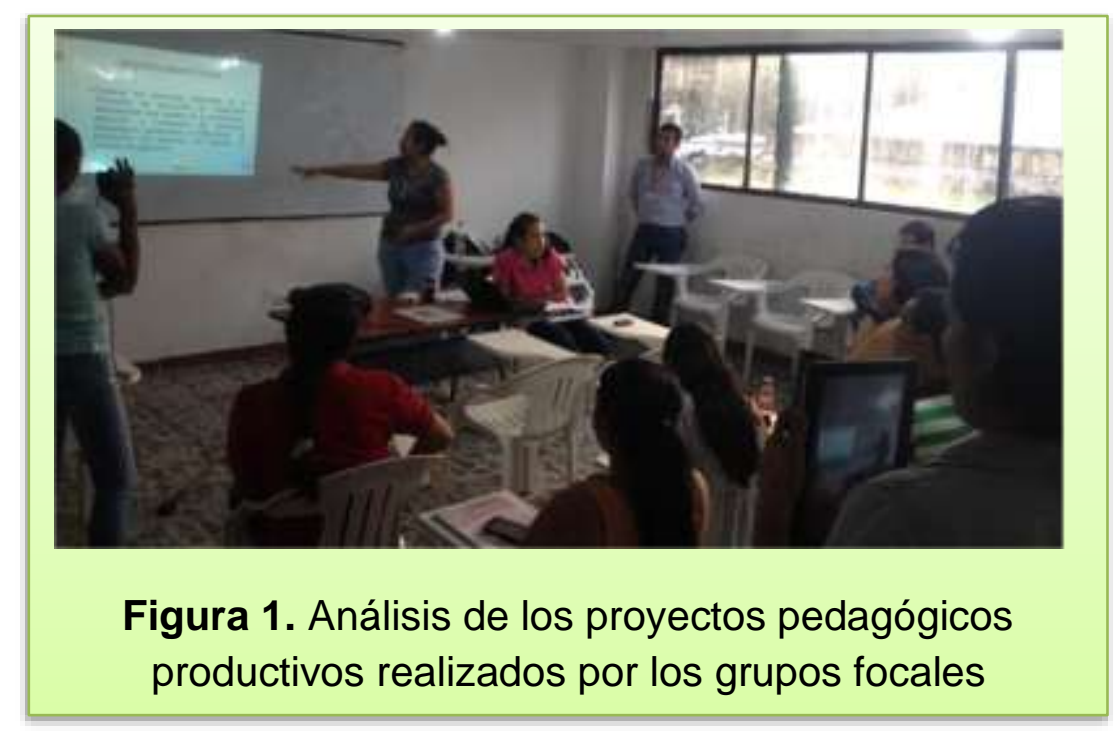

\section{RESULTADOS Y DISCUSIÓN}

La participación de las instituciones educativas en los PPP por municipio, se detalla en la Gráfica 1, siendo la de mayor participación Macanal en San Juan de Arama con el 27\%. De total de los PPP el 65\% pertenecen a la línea agrícola; en el análisis de la información recolectada se encontró que esto se debe en gran parte, a que, en el momento de la llegada del practicante, las instituciones educativas se encuentran adelantando contenidos relacionados con el área agrícola; además disponen de amplios terrenos para el establecimiento de los cultivos. También se puede influir la seguridad, puesto que hay menor riesgo de pérdidas en un proyecto agrícola, y posiblemente la infraestructura, equipos y materiales disponibles también influyeron a la hora de seleccionar el PPP.

Por otro lado, la mayoría de las instituciones educativas no contaban con recursos suficientes para la implementación de propuestas con especies pecuarias, siendo más económico y de mayor facilidad la consecución de los insumos para implementar los proyectos agrícolas, teniendo en cuenta el interés del practicante, asesor y de la institución. 


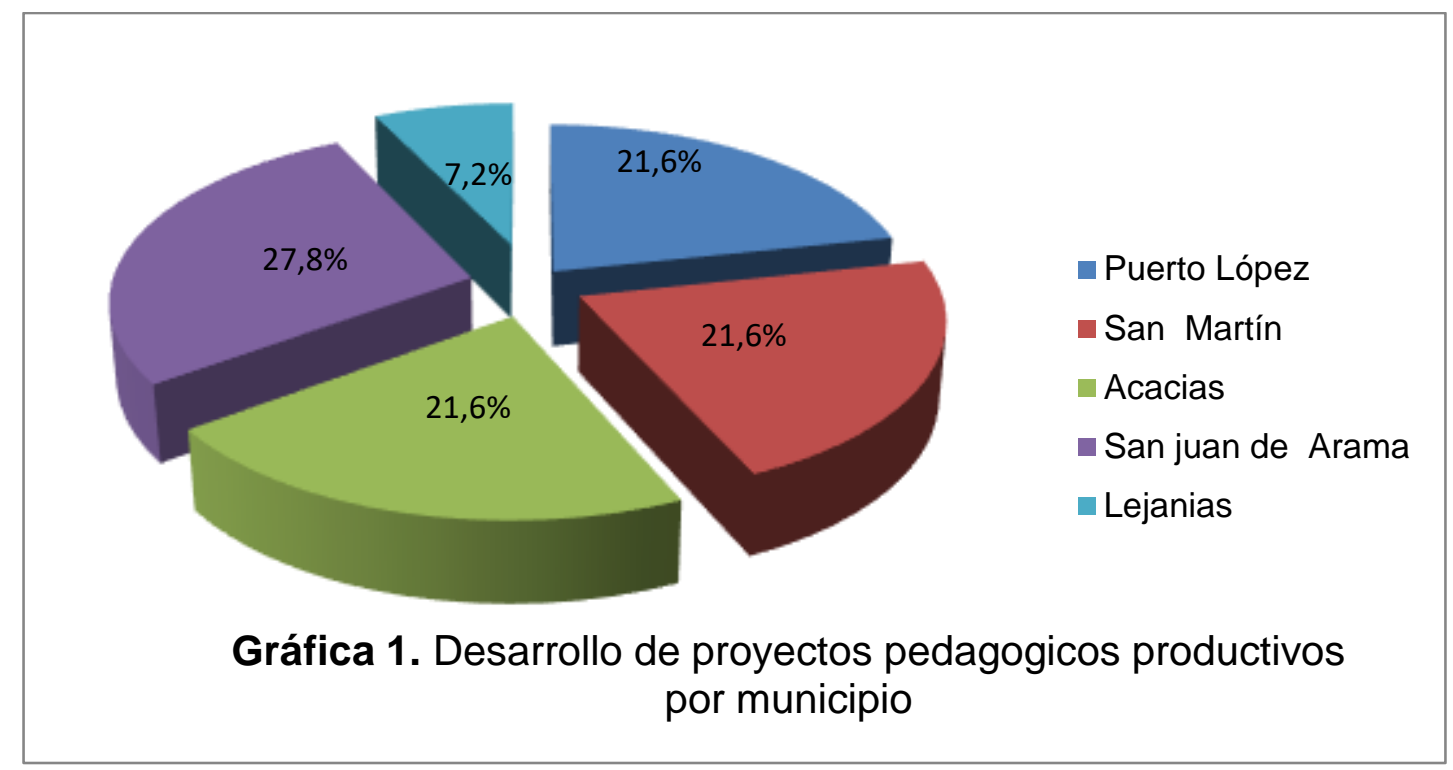

Las temáticas en las cuales se desarrollaron los PPP de la línea agrícola fueron: huertas con plantas medicinales, laboratorio artesanal para la producción de harina de yuca, huertas de hortalizas orgánicas, viveros, plantas ornamentales, cultivo de plátano, buenas prácticas ambientales, abonos orgánicos y biopreparados, técnicas de producción agrícola ecológica sostenible, policultivos de moringa-plátano y ahuyama, granja agropecuaria de autoconsumo, cítricos, bancos de proteínas con árboles, pasto de corte y ensilaje, programas de seguridad alimentaria, caña de azúcar, maracuyá y papa aérea.

En cuanto a los proyectos desarrollados en la línea pecuaria el porcentaje arrojado fue del $25 \%$, donde se evidencian el interés del estudiante de la Universidad por cuanto influye en gran medida la línea de profundización a la que perteneció, también se encontró en algunas instituciones educativas un interés institucional, con el fin de optimizar las instalaciones y apoyo financiero para la ejecución de proyectos pecuarios.

El 10\% restante de los PPP en extensión lo realizaron instituciones de educación no formal o dedicadas al desarrollo y al trabajo humano, como asociaciones de productores, madres comunitarias y personas en condición de desplazamiento. Con quienes se realizaron proyectos de emprendimiento, comercialización de productos, procesos de seguridad alimentaria y educación ambiental. Este análisis da 
cumplimiento a lo establecido en la ley 115 de 1994 que plantea la educación no formal, como estrategia que conlleva al desarrollo comunitario, y además se confirma que la pedagogía permite la interacción entre los referentes teóricos y conceptuales apropiados y reflexionados en los diferentes contextos donde se desarrollan los procesos educativos.

Con relación a la evidencia de los aportes que desde los PPP se han hecho a las modalidades de educación agropecuaria, los resultados se sustentan en la acción misma de los practicantes de UNILLANOS, en tanto que algunos han hecho aportes desde lo curricular, con propuestas para fortalecer los contenidos temáticos, otros a partir de su gestión y liderazgo han logrado establecer alianzas con entidades del orden público y privado, permitiendo mejorar los procesos y dejando puertas abiertas para adelantar otros proyectos en las instituciones, así mismo, se ha fortalecido desde la incorporación de nuevas herramientas tecnológicas, el rescate de la parte cultural, la inclusión del desarrollo tecnológico, la tecnificación de procesos, logrando promover el emprendimiento en los estudiantes, incentivando la curiosidad a partir de experiencias reales, contribuyendo con la generación de conciencia en los estudiantes para la implementación de la producción agroecológica de alimentos a partir de la los sistemas de producción en sus granjas, aplicando prácticas para la conservación de especies agrícolas, procesos de seguridad alimentaria, y por último y no menos importante, mediante las respuestas de los estudiantes de las instituciones también se evidencio que, por medio de los PPP se impulsó el trabajo en equipo mediante la unión de los docentes y los estudiantes.

Referente a la vinculación de los miembros de las comunidades educativas, la participación de los estudiantes fue del $74 \%$, y el de los asesores fue de $11 \%$, en el diseño, ejecución y evaluación de los PPP, pero el estudio muestra como una gran dificultad la vinculación de padres de familia (participación del 15\%) (Gráfica 2). En instituciones educativas que cuentan con internados, especialmente presentaron mayores inconvenientes porque ellos asisten solo algunos fines de semana para visitar a sus hijos y para la entrega de informes académicos, lo cual dificulta la 
interacción con el practicante, especialmente si éste no se encuentra interno; situación contraria sucede con los padres de familia que viven en cercanía a la institución educativa, puesto que allí se encontró un apoyo interesante interactuando con el practicante en el desarrollo del proyecto, haciendo que el impacto transcendiera hacia los padres y se aprovechara el conocimiento para el progreso familiar.

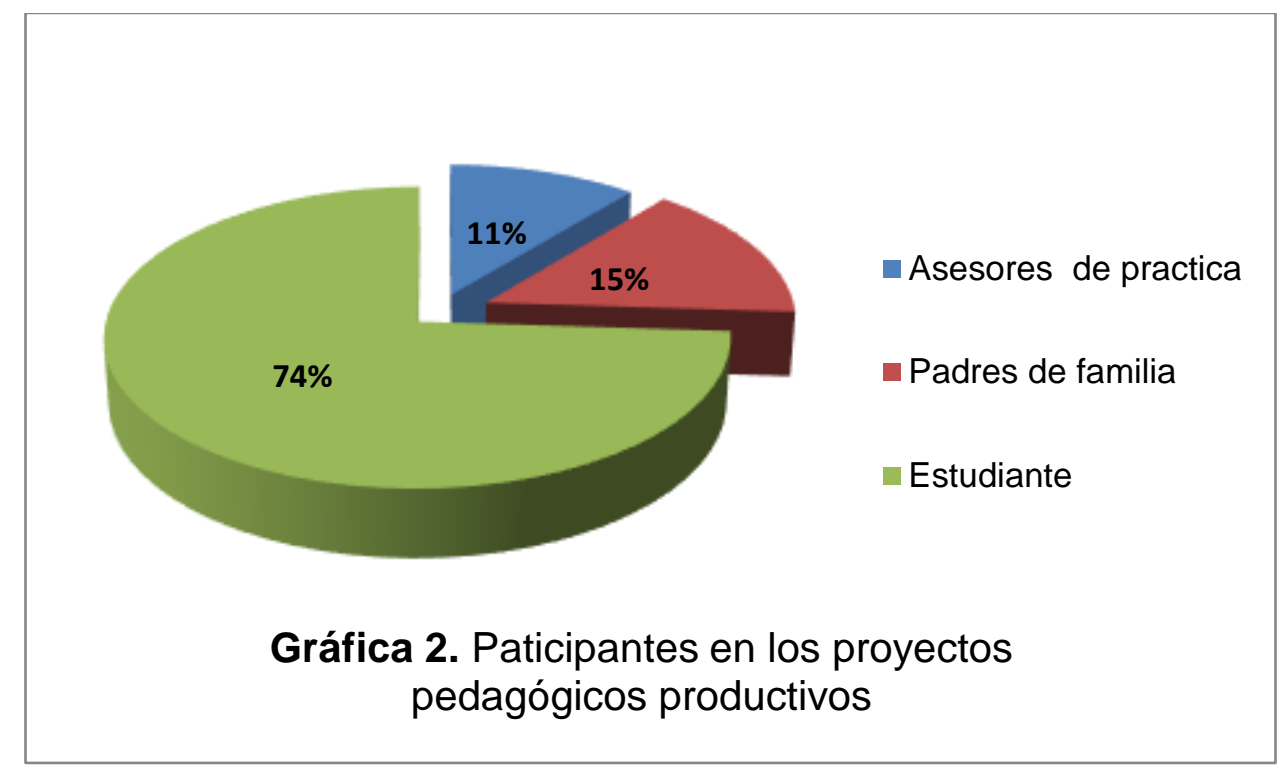

Por su parte los asesores no solo participaron de la selección, diseño y ejecución de los PPP, sino que además en muchas ocasiones imponen el problema de investigación, lo que obedece a un interés personal, interés institucional, o del área, lo que puede ser uno de los argumentos que hace posible la continuidad del proyecto en las instituciones, esta afirmación refleja la importancia que tienen los PPP como herramienta didáctica para el fortalecimiento del proceso enseñanzaaprendizaje tal y como lo plantea la Ley 115 de 1994 (CRC, 1994).

Algunos estudiantes integrados en los PPP cuando logran ser motivados por el practicante replican el proyecto en sus familias y en sus proyectos de vida, cuando no sucede así, los proyectos como ellos lo manifestaron desaparecen porque no se generó identidad y apropiación. Contrario a lo anterior para los PPP que se desarrollaron en instituciones de educación no formal, se encontró que la vinculación se realizó de manera voluntaria y motivada desde las entidades hacia 
sus usuarios, siendo el papel del practicante fortalecer esa motivación y sembrar en los miembros de las comunidades habilidades innovadoras que fueron las de mayor impacto (40\%) en comparación con las emprendedoras y tecnificación de procesos, siendo su impacto del 30\% (Gráfica 3).

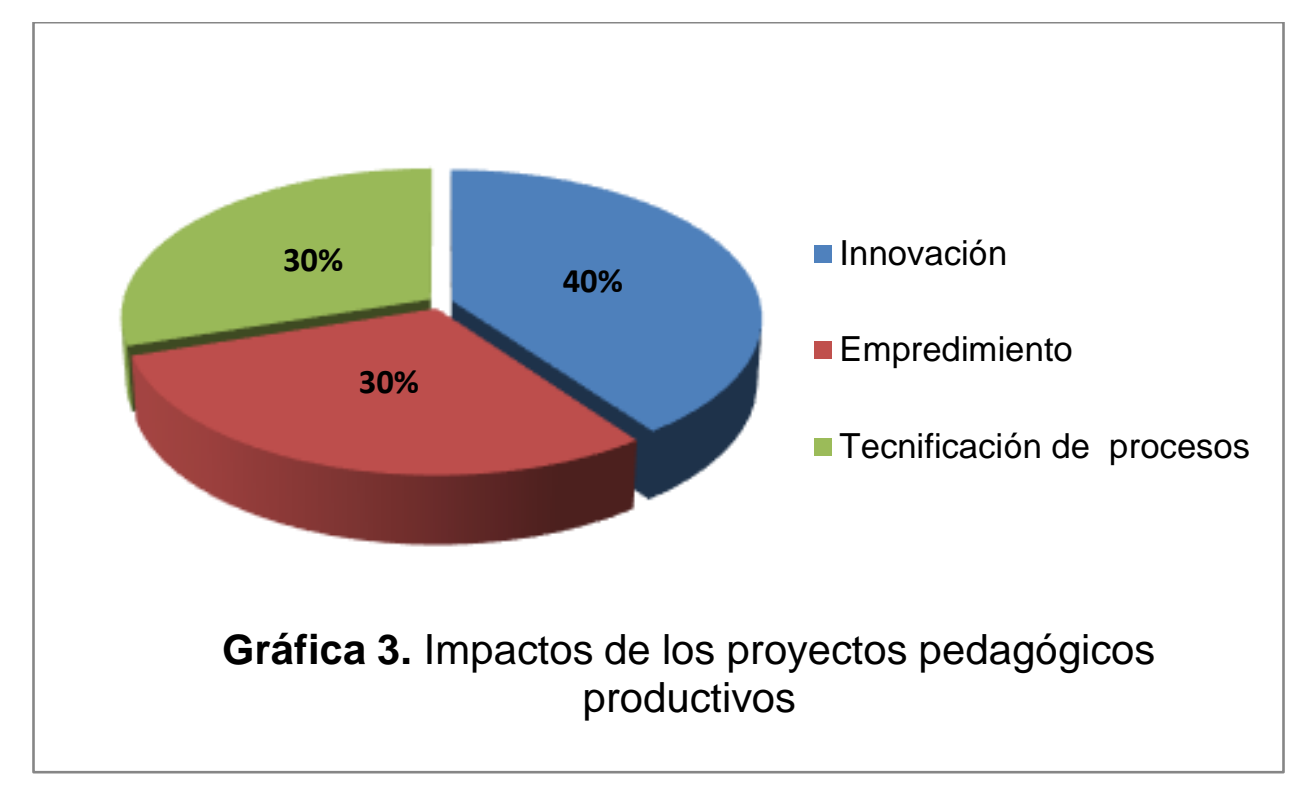

Referente a las respuestas de las preguntas de la encuesta relacionadas con el impacto de los PPP (Cuadro 1), es importante señalar que la enseñanza ha ayudado a reforzar el conocimiento mediante la práctica, estimulando la investigación aplicada para la solución de problemas agropecuarios y en ocasiones algunas familias de los estudiantes han obtenido ingresos adicionales por este proceso.

En el marco de los convenios de cooperación interinstitucional, el equipo investigador encontró que las instituciones educativas han mostrado poco compromiso para lograr la continuidad de los PPP dentro del proceso educativo, lo que refleja un bajo impacto en las competencias de los cursos que fueron involucrados estos proyectos, porque con el tiempo desaparecen sin que sea posible evidenciar la sostenibilidad y fortalecimiento de las competencias en los estudiantes por parte de los docentes de la institución.

Por último, para los hoy egresados del programa de LPA, practicantes en el periodo analizado, ellos consideran que el los PPP han contribuido para mejorar la calidad de vida de las comunidades educativas, y desde la perspectiva personal, el diseñar, 
ejecutar y evaluar estos proyectos fue una gran oportunidad que les permitió experimentar e innovar, y que esta experiencia ha trascendido a su vida laboral. Estas afirmaciones permiten evidenciar el cumplimiento del propósito de formación plateado por el programa, que literalmente argumenta que formar un maestro con alto contenido social, humanístico, pedagógico y científico es capaz de trasformar la realidad y liderar procesos en lo educativo y en lo agropecuario.

Cuadro 1. Respuestas a las preguntas relacionadas con el impacto de los proyectos pedagógicos productivos

\begin{tabular}{|c|c|}
\hline Preguntas de la encuesta & Respuestas de los encuestados \\
\hline \multirow{5}{*}{$\begin{array}{l}\text { ¿Qué aspectos positivos } \\
\text { encuentra usted en los } \\
\text { proyectos pedagógicos } \\
\text { productivas desarrollados } \\
\text { por los practicantes? }\end{array}$} & $\begin{array}{l}\text { "Nos enseñan sobre la utilización de los recursos } \\
\text { en pro de las actividades de campo; se pone a } \\
\text { prueba los conocimientos en lo práctico" }\end{array}$ \\
\hline & $\begin{array}{l}\text { "Orientan procesos de investigación y desarrollar } \\
\text { y reforzar el aprendizaje." }\end{array}$ \\
\hline & $\begin{array}{l}\text { "Traen ideas nuevas para el desarrollo de los } \\
\text { proyectos pedagógico productivo en la institución } \\
\text { educativa" }\end{array}$ \\
\hline & $\begin{array}{l}\text { "Los estudiantes ven otras oportunidades de } \\
\text { aprendizaje a través de los proyectos } \\
\text { pedagógicos productivos". }\end{array}$ \\
\hline & $\begin{array}{l}\text { "El proyecto pedagógico productivo permite } \\
\text { generar ingresos a la comunidad a través de la } \\
\text { elaboración de productos". }\end{array}$ \\
\hline \multirow{3}{*}{$\begin{array}{l}\text { ¿Considera que los } \\
\text { proyectos ejecutados por } \\
\text { los practicantes han sido } \\
\text { pertinentes? }\end{array}$} & $\begin{array}{l}\text { "Si, responden a una problemática o necesidad } \\
\text { de la comunidad". }\end{array}$ \\
\hline & $\begin{array}{l}\text { "Si, por que los estudiantes profundizan } \\
\text { temáticas y desarrollan diferentes tipos de } \\
\text { aprendizaje". }\end{array}$ \\
\hline & $\begin{array}{l}\text { "Si, por que dan a conocer de manera práctica los } \\
\text { conocimientos a través de la investigación e } \\
\text { innovación". }\end{array}$ \\
\hline \multirow{4}{*}{$\begin{array}{c}\text { ¿La realización del } \\
\text { proyecto pedagógico } \\
\text { productivo dejó a la } \\
\text { institución algún valor } \\
\text { agregado? }\end{array}$} & $\begin{array}{l}\text { "Procesos de investigación y conocimientos } \\
\text { innovadores". }\end{array}$ \\
\hline & "Convenios y alianzas con otras instituciones" \\
\hline & "Recursos". \\
\hline & "Posibilidad de becas para algunos estudiantes". \\
\hline
\end{tabular}




\section{CONCLUSIONES}

La caracterización de los proyectos pedagógicos productivos desarrollados en la práctica profesional docente por estudiantes del programa LPA de UNILLANOS durante un periodo de tres años evidencian que los actores involucrados en el diseño, ejecución y evaluación de los mismos fueron estudiantes de las instituciones educativas, asesores y padres de familia, debido al desarrollo del objetivo pedagógico que involucra acciones curriculares y que en la mayoría de los casos fortalecen contenidos temáticos de la modalidad agropecuaria, por lo tanto, su participación fue dinámica para el éxito de los PPP.

El estudio permitió evidenciar que los PPP desarrollados han incidido en el mejoramiento de la calidad de vida de algunas familias, permitiendo ofrecer alternativas de seguridad alimentaria y el fortalecimiento de los procesos productivos en las instituciones.

Los practicantes concluyeron que el ejercicio de la práctica profesional docente permite desarrollar destrezas, habilidades, conocimientos académicos, y personales que, en términos del diseño, ejecución y evaluación de los PPP, los preparan para abordar retos el campo laboral.

\section{RECOMENDACIONES}

Se recomienda ampliar periodo de la práctica profesional docente en el cual los practicantes tengan suficiente tiempo para ejecutar con la comunidad los PPP en todas sus etapas: diagnóstico, planeación y ejecución.

Se recomienda a las instituciones educativas dar mayor importancia y acompañamiento económico para el desarrollo de los PPP realizados por los estudiantes de UNILLANOS, con el fin de fortalecer sus prácticas docentes y así mismo, de esta manera apoyar la consecución de proyectos, fortaleciendo, los convenios que se tienen con otras instituciones. 


\section{REFERENCIAS BIBLIOGRÁFICAS}

1. Alcaldía Local de Acacias (ALA). Plan de desarrollo municipal "Para vivir bien" 2016-2019 Alcaldía de Acacias, Acacias, Colombia. 219 p. 2016.

2. Alcaldía Local de Puerto López (ALPL). Informe de gestión "Puerto López para todos 2016-2019". Alcaldía de Puerto López, Puerto López, Colombia. 55 p. 2017.

3. Alcaldía Local de San Martin de los Llanos (ALSM). Municipio de San Martin de los Llanos. Plan de desarrollo "Por el San Martin que queremos" 2004-2007. Alcaldía de San Martin, San Martin, Colombia. 54 p. 2004.

4. Alcaldía Local de San Martin de los Llanos (ALSM). Municipio de San Martin de los Llanos. Plan de desarrollo "La nueva fuerza del cambio" 2012-2015. Alcaldía de San Martin, San Martin, Colombia. 123 p. 2012.

5. Alcaldía Local de Lejanías (ALL). Plan de desarrollo del Municipio de Lejanías 2016-2019 "Compromiso serio". Lejanías, Colombia. 191 p. 2016.

6. Becerra G.Y. Los proyectos pedagógicos de aula y la globalización. Educere. 8 (24): 111-118. 2004.

7. Consejo Municipal de San Juan de Arama (CMSJA). Acuerdo No. 004 "Por el cual se adopta el plan de desarrollo para el municipio de San Juan de Arama, Meta 2016-2019, Oportunidades con equidad social" Municipio de San Juan de Arama, San Juan de Arama, Colombia. 44 p. 2016.

8. Congreso de la República de Colombia (CRC). Ley 115 de Febrero 8 de 1994 "Por la cual se expide la ley general de educación". Congreso de la República de Colombia, Bogotá, Colombia. 50 p. 1994.

9. Fundación Manuel Mejía (FMM). Los proyectos pedagógicos productivos (PPP) y el desarrollo de competencias. Ministerio de Educación Nacional, Bogotá, Colombia. 34 p. 2010.

10. Gobernación del Valle del Cauca y Universidad del Valle (GVC, UV). Estrategias y propuestas de educación y pedagogía rural: Nuevas opciones para la nueva ruralidad. Universidad del Valle, Cali, Colombia. 177 p. 2013.

11. Herrera J.L. La formación de los profesionales universitarios en las empresas en el contexto cubano. Revista ieRed: Revista Electrónica de la Red de Investigación Educativa. 1 (4): 1-18. 2006.

12. Martínez M. Formación para la ciudadanía y educación superior. Revista Iberoamericana de Educación. 42: 85-102.

13. Martínez M., Esteban F. Una propuesta de formación ciudadana para el EEES. Revista Española de Pedagogía. 63 (230): 63-83. 2005.

14. Ministerio de Educación Nacional (MEN). Introducción a los PPP. Ministerio de Educación Nacional, Bogotá, Colombia. 30 p. 2010a.

15. Ministerio de Educación Nacional (MEN). Manual de implementación caminar en secundaria. Estrategia para la nivelación de los estudiantes en extra edad de básica secundaria en establecimientos educativos del sector rural. Ministerio de Educación Nacional, Bogotá, Colombia. 112 p. 2010 b.

16. Ministerio de Educación Nacional (MEN). Los proyectos pedagógicos productivos, estrategias para el mejoramiento de la calidad de la educación. (Informe final: Contrato OIM-PS 7123, Evaluación del acompañamiento 
realizado a establecimientos educativos e instituciones educativas para el desarrollo de proyectos pedagógicos productivos - PPP). Ministerio de Educación Nacional, Bogotá, Colombia. 121 p. 2012a.

17. Ministerio de Educación Nacional (MEN). Proyectos pedagógicos productivos. Orientaciones para estudiantes. Ministerio de Educación Nacional, Bogotá, Colombia. 85 p. $2012 b$.

18. Moreno J.J., Anaya S.L., Benavides P., Hernández U. Los proyectos pedagógicos de aula para la Integración de las TIC, Convenio entre la Universidad del Cauca y Computadores para Educar Facultad de Ingeniería Electrónica y Telecomunicaciones Departamento de Sistemas, 48 p. 2010. Recuperado 17 Diciembre $2017 . \quad$ Disponible En: www.ciudadaniadigital.gov.co/627/articles-12649 recurso PDF.pdf

19. Ortíz A.L. Modelos pedagógicos y teorías del aprendizaje. ¿Cómo elaborar el modelo pedagógico de la institución educativa? Ediciones de la U, 160 p. 2013.

20. Pacheco J.C., Gómez G., Barrero G. El desafío de las comunidades artesanales rurales: una propuesta ecotecnológica para una artesanía sostenible. Acta Agronómica. 58 (3): 206-220. 2009.

21. Sabino C. El proceso de investigación. Editorial Episteme, Guatemala. 240 p. 2014. 